\title{
Obesity Impacts Mortality and Rate of Revascularizations Among Patients With Acute Myocardial Infarction: An Analysis of the National Inpatient Sample
}

\author{
Genaro Velazquez ${ }^{1}$, Trisha Marie A. Gomez ${ }^{1}$, Iriagbonse Asemota ${ }^{1}$, Emmanuel Akuna ${ }^{1}$, Pius E. Ojemolon \\ 2 , Precious Eseaton ${ }^{3}$ \\ 1. Internal Medicine, John H. Stroger, Jr. Hospital of Cook County, Chicago, USA 2. Anatomical Sciences, St. George's \\ University, St. George's, GRD 3. Internal Medicine, College of Medicine, University of Benin, Benin City, NGA
}

Corresponding author: Precious Eseaton, precious.eseaton@yahoo.com

\section{Abstract \\ Background}

Obesity is now a recognized chronic comorbid condition which is highly prevalent in the United States. Obesity poses several health risks, affecting multiple organ systems. The cardiovascular system is particularly affected by obesity including its role in atherosclerotic disease and hence myocardial infarction (MI) from atheromatous plaque events. However, multiple population-based studies have shown mixed outcomes in obese patients who have acute MI. This study aimed to determine if obesity paradoxically improved outcomes in patients with acute myocardial infarction (AMI) as well as compare outcomes of mild to moderately obese patients and morbidly obese patients to non-obese patients.

\section{Materials and methods}

Data was obtained from the Nationwide Inpatient Sample (NIS) for 2016 and 2017. The study included adult patients with a principal discharge diagnosis of AMI. This group was divided into ST segment elevation myocardial infarction (STEMI) and non-ST segment myocardial infarction (NSTEMI). Obese patients were subdivided into two groups: mild-moderate obesity and morbid obesity. Primary outcome compared inpatient mortality. Secondary outcomes included rate of percutaneous coronary intervention (PCI), coronary artery bypass grafting (CABG), composite revascularization, mean length of hospitalization, total hospital charges, and rates of complications.

\section{Results}

In patients with STEMI, mild to moderately obese patients had lower odds of mortality (aOR: $0.80,95 \% \mathrm{CI}$ : $0.715-0.906, \mathrm{p}<0.001$ ) compared to non-obese patients. However, morbidly obese patients had higher odds of mortality (aOR: 1.26, 95\% CI: 1.100-1.446, p < 0.001) compared to non-obese patients. Mild to moderately obese patients had higher odds of composite revascularization (aOR: 1.24, 95\% CI: 1.158-1.334, p < 0.001), PCI (aOR: 1.08, 95\% CI: 1.054-1.150, p = 0.014), and CABG (aOR: 1.46, 95\% CI: 1.313-1.626, p < 0.001).

Review began $11 / 16 / 2020$ Review ended 11/26/2020 Published 12/04/2020

\section{() Copyright 2020}

Velazquez et al. This is an open access article distributed under the terms of the Creative Commons Attribution License CC-BY 4.0., which permits unrestricted use, distribution, and reproduction in any medium, provided the original author and source are credited.

\section{Conclusion}

The degree of obesity affects outcome of patients with AMI. Cardiovascular interventions during hospitalizations for AMI also varied with degree of obesity. This may have affected the outcome, especially among morbidly obese patients.

Categories: Cardiology, Endocrinology/Diabetes/Metabolism, Internal Medicine

Keywords: obesity, obesity paradox, acute myocardial infarction, nis, cardiovascular disease

\section{Introduction}

Obesity is now a recognized chronic comorbid condition which is highly prevalent in the United States. It has a higher prevalence among the middle age female population $[1,2]$. Obesity poses several health risks, affecting multiple organ systems [3, 4]. The cardiovascular system is particularly affected by obesity. The mechanism through which obesity affects the cardiovascular system includes adipokine dysregulation, inflammation, increased circulating free fatty acids, increased oxidative stress and adipose tissue hypoxia, ultimately contributing to atherosclerosis and the development of atheromatous plaques [5]. The development and subsequent disruption of atheromatous plaques results in atherothrombosis, which is the hallmark of acute myocardial infarction (AMI) [6]. Studies involving outcomes of AMI in obese population have yielded mixed results [7-11]. Improved outcomes have fueled concepts including metabolically healthy obesity and the obesity paradox relating to cardiovascular diseases. It is also suggested that this paradox may be due to unaccounted confounding factors yet to be objectively identified [12]. This study aimed to 
determine if obesity paradoxically improved outcomes in patients with AMI as well as compare outcomes of mild to moderately obese patients and morbidly obese patients to non-obese patients.

\section{Materials And Methods \\ Design and data source}

This study was a retrospective cohort study involving adult patients (aged $\geqslant 18$ years) hospitalized for AMI in the US between January 1, 2016 and December 31, 2017. Data was obtained from the Nationwide Inpatient Sample (NIS) database for 2016 and 2017. The NIS is a database of hospital inpatient stays derived from billing data submitted by hospitals to statewide data organizations across the US, covering more than $97 \%$ of the US population [13-16]. It approximates a $20 \%$ stratified sample of discharges from US community hospitals, excluding rehabilitation and long-term acute care hospitals [17,18]. This dataset is weighted to obtain national estimates $[19,20]$. Both the 2016 and 2017 database are coded using the International Classification of Diseases, Tenth Revision, Clinical Modification/Procedure Coding System (ICD-10-CM/PCS) $[21,22]$.

\section{Study population}

The study included adult patients with a principal discharge diagnosis of AMI $(n=1,299,885)$. This group was divided into ST segment elevation myocardial infarction (STEMI: I21.0, I21.1, I21.2, I21.3) and non-ST segment myocardial infarction (NSTEMI: I21.4). A total of 349,900 hospitalizations were for STEMI, out of which $16.5 \%(n=57,734)$ were obese and $83.5 \%(n=292,166)$ were non-obese. A total of 949,985 hospitalization were for NSTEMI, out of which $19.8 \%(n=188,097)$ were obese and $80.2 \%(n=761,888)$ were non-obese (Figure 1). Patients were excluded if STEMI or NSTEMI was a secondary diagnosis or if they developed AMI following a procedure. The cohort of patients with AMI was further divided based on the presence of a secondary discharge diagnosis of obesity (E66.0, E66.1, E66.2, E66.8, E66.9, Z68.3, Z68.4). We combined both general codes for obesity as well as BMI specific codes to accurately capture obesity categories. Obese patients were subdivided into two groups: mild-moderate obesity and morbid obesity, using the above codes, correlating with a BMI of 30-39 and 40 and above, respectively. The ICD-10-CM/PCS codes used to obtain the cohort can be found in the Appendix.

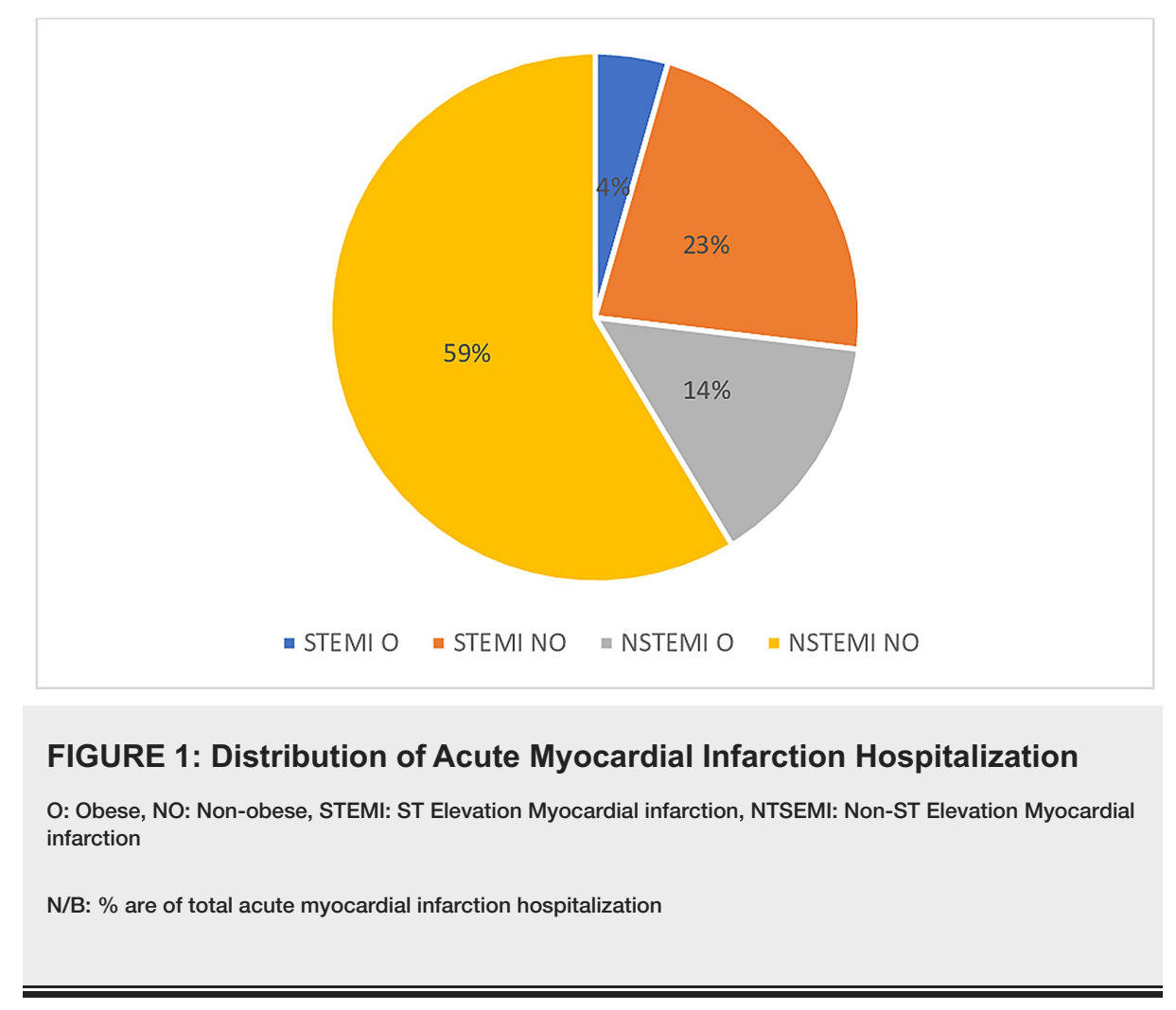

\section{Outcome measures}

The primary outcome was comparing inpatient mortality among patients with AMI based on presence or absence of obesity. Secondary outcomes in this population included need for percutaneous coronary intervention (PCI) with drug eluting stent and bare metal stent placement, coronary artery bypass grafting $(\mathrm{CABG})$, and composite revascularization (PCI and $\mathrm{CABG}$ ). Other outcomes included rate of complications including need for electrical cardioversion/defibrillation and odds of having a secondary discharge diagnosis of acute kidney failure (AKI) and cardiogenic shock. We also compared mean length of hospitalization as well 
as total hospital charges between both groups as measures of healthcare utilization cost.

\section{Statistical analysis}

Data was analyzed using Stata ${ }^{\circledR}$ (Statistics and Data) Version 16 software (StataCorp, Texas, USA). All analyses were conducted using the weighted samples for national estimates in adjunct with Healthcare Cost and Utilization Project regulations for using the NIS database. Co-morbidities were calculated as proportions of the cohort and Chi squared test was used to compare association between the non-obese and the obese subgroups. Multivariate regression analysis was done to adjust for possible confounders while calculating the primary and secondary outcomes. The patient and hospital characteristics as well as comorbidities were obtained during literature review. A univariate screen was done to further confirm these factors. Variables with $\mathrm{p}<0.2$ in univariate screen were included in multivariable regression model. A p-value of 0.05 was set as the threshold for statistical significance in the multivariate regression analysis.

\section{Ethical considerations}

The NIS database does not contain patient identifiers. Since 2012, the NIS has also removed state level and hospital identifiers. This has enhanced patient protection and anonymity. This study was exempt from Institutional Review Board approval.

\section{Results}

\section{Characteristics of STEMI patients}

Data showed 349,900 hospitalizations were for STEMI during the study period. The prevalence of obesity among patients with STEMI was $16.5 \%$.

Obese patients were significantly younger ( 59.2 vs 64.3 years, $\mathrm{p}<0.001$ ), with a higher proportion of females (34.4 vs 30.2\%, $\mathrm{p}<0.001$ ). Compared to non-obese patients, obese patients had a higher proportion with comorbidities including hypertension ( 57.3 vs $50.7 \%, \mathrm{p}<0.001$ ), diabetes ( 47.9 vs $28.0 \%$, p < 0.001), smoking history ( 52.5 vs $51.4 \%, \mathrm{p}=0.034)$, congestive heart failure ( 25.7 vs $23.0 \%, \mathrm{p}<0.001)$ and chronic kidney disease $(10.5$ vs $8.5 \%, \mathrm{p}<0.001)$ (Table 1$)$.

\begin{tabular}{|c|c|c|c|c|c|c|}
\hline \multirow{2}{*}{ Variable } & & STEMI N = 349,900 & & & NSTEMI N = 949,985 & \multirow[b]{2}{*}{$\mathrm{p}$-value } \\
\hline & Obese, \% & Non-obese, \% & $\mathrm{p}$-value & Obese, \% & Non-obese, \% & \\
\hline \multicolumn{7}{|c|}{ Patient characteristics } \\
\hline Percent & 16.5 & 83.5 & & 19.8 & 80.2 & \\
\hline Mean Age, years & 59.2 & 64.3 & $<0.001$ & 63.0 & 69.5 & $<0.001$ \\
\hline Females & 34.4 & 30.2 & $<0.001$ & 42.8 & 40.1 & $<0.001$ \\
\hline \multicolumn{2}{|l|}{ Racial distribution } & & $<0.001$ & & & $<0.001$ \\
\hline White & 73.1 & 72.0 & & 70.2 & 70.5 & \\
\hline Black & 9.4 & 8.2 & & 13.4 & 11.4 & \\
\hline Hispanic & 8.2 & 7.8 & & 8.6 & 8.1 & \\
\hline Others & 9.3 & 12.0 & & 7.8 & 10.0 & \\
\hline \multicolumn{2}{|l|}{ Insurance type } & & $<0.001$ & & & $<0.001$ \\
\hline Medicaid & 37.4 & 48.5 & & 54.2 & 66.3 & \\
\hline Medicare & 12.8 & 10.6 & & 11.3 & 8.7 & \\
\hline Private & 42.6 & 34.1 & & 30.0 & 21.4 & \\
\hline Uninsured & 7.2 & 6.8 & & 4.5 & 3.6 & \\
\hline \multicolumn{3}{|c|}{ Charlson Comorbidity Index score } & $<0.001$ & & & $<0.001$ \\
\hline 1 & 25.7 & 34.7 & & 20.5 & 21.6 & \\
\hline 2 & 32.4 & 30.8 & & 22.5 & 20.5 & \\
\hline$\geq 3$ & 41.9 & 34.5 & & 57.0 & 55.9 & \\
\hline
\end{tabular}




\section{Cureus}

Median annual income expected for patient's zip code, US\$

$<0.001$

$<0.001$

$\begin{array}{lllll}1-43,999 & 28.6 & 28.5 & 32.7 & 31.3 \\ 44,000-55,999 & 28.2 & 27.5 & 28.1 & 27.4 \\ 56,000-73,999 & 25.0 & 23.9 & 23.4 & 23.0 \\ \geq 74,000 & 18.2 & 20.1 & 15.8 & 18.3\end{array}$

Comorbidities*

Hypertension

$<0.001 \quad 47.6$

$<0.001$

Diabetes

Smoking history

47.9

$<0.001 \quad 60.2$

$<0.001$

0.034

$<0.001$

Atrial fibrillation/flutter

$14.1 \quad 14.4$

$0.372 \quad 20.6$

$<0.001$

CHF

25.7

$<0.001$

39.6

$<0.001$

CKD

10.5

$<0.001 \quad 23.8$

$<0.001$

Dialysis dependence

$1.2 \quad 1.1$

$0.643 \quad 3.5$

$<0.001$

Dyslipidemia

$72.4 \quad 61.3$

$<0.001 \quad 75.3$

$<0.001$

Chronic IHD

$87.1 \quad 84.2$

$<0.001 \quad 83.1$

$<0.001$

Old PCI

$1.4 \quad 1.5$

$0.874 \quad 1.7$

0.148

Old CABG

$4.1 \quad 4.5$

$0.102 \quad 10.6$

$<0.001$

Pacemaker

0.8

$<0.001 \quad 2.5$

$<0.001$

Prior CVA

Liver disease

1.1

0.11

$<0.001$

$0.012 \quad 3.6$

$<0.001$

COPD

4.8

4.3

$0.103 \quad 21.6$

$<0.001$

Supplemental $\mathrm{O}_{2}$

$12.2 \quad 11.7$

$<0.001 \quad 3.7$

$<0.001$

Hypothyroidism

$1.3 \quad 0.9$

$0.045 \quad 13.3$

0.171

Electrolyte disorders

$9.0 \quad 8.4$

$0.186 \quad 22.1$

0.837

$\begin{array}{lll}0.004 & 26.2 & 25.7\end{array}$

0.040

Hospital characteristics

Hospital region

$<0.001$

$<0.001$

Northeast

$15.8 \quad 17.1$

Midwest

25.8

22.2

South

39.8

40.6

West

18.6

20.1

Hospital bed size

(2)

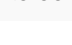

$16.0 \quad 18.6$

$25.2 \quad 21.7$

$40.8 \quad 41.0$

$18.0 \quad 18.7$

0.462

0.030

$\begin{array}{lcc}\text { Small } & 15.1 & 14.5 \\ \text { Medium } & 29.6 & 29.8 \\ \text { Large } & 55.3 & 55.7 \\ \text { Urban location } & 94.3 & 93.3 \\ \text { Teaching hospital } & 68.8 & 67.6\end{array}$

$\begin{array}{rrr} & 16.6 & 17.4 \\ & 30.5 & 30.8 \\ & 52.9 & 51.8 \\ <0.001 & 93.0 & 91.6 \\ 0.035 & 68.0 & 65.6\end{array}$

$<0.001$

$<0.001$ 


\section{Cureus}

\section{TABLE 1: Patient and hospital characteristics of STEMI and NSTEMI by obesity}

\#: For 2017; *: Secondary diagnosis

CABG: Coronary artery bypass grafting, CHF: Congestive heart failure, CKD: Chronic kidney disease, COPD: Chronic obstructive pulmonary disease, CVA: Cerebrovascular accident, IHD: Ischemic heart disease, NSTEMI: Non-ST segment elevation myocardial infarction, PCI: Percutaneous coronary intervention, STEMI: ST segment elevation myocardial infarction.

\section{Primary outcome in STEMI patients: in-hospital mortality}

The in-hospital mortality for patients with STEMI was $8.0 \%$ overall. Mild to moderately obese patients had lower odds of mortality (aOR: $0.80,95 \%$ CI: 0.715-0.906, p < 0.001) compared to non-obese patients. However, morbidly obese patients had higher odds of mortality (aOR: $1.26,95 \%$ CI: $1.100-1.446, \mathrm{p}<0.001$ ) compared to non-obese patients (Tables 2, 3).

\begin{tabular}{|c|c|c|c|c|}
\hline Outcome & Mild-moderate, \% & Nonobese, \% & aOR (95\% Cl) & p-value \\
\hline \multicolumn{5}{|l|}{ Primary outcome } \\
\hline In-hospital mortality & 5.1 & 8.4 & $0.80(0.715-0.906)$ & $<0.001^{*}$ \\
\hline \multicolumn{5}{|l|}{ Secondary outcomes } \\
\hline Mean Length of stay, days $(95 \% \mathrm{Cl})$ & $4.1(3.9-4.2)$ & $4.0(4.0-4.1)$ & $0.00(-0.107-0.102)$ & 0.965 \\
\hline Mean total hospital charges, US\$ (95\% Cl) & $113000(109300-116600)$ & $107600(105400-109800)$ & $2200(-800-5100)$ & 0.151 \\
\hline PCl with DES & 69.9 & 65.1 & $1.12(1.054-1.180)$ & $<0.001^{*}$ \\
\hline $\mathrm{PCl}$ with BMS & 8.6 & 9.2 & $0.93(0.851-1.023)$ & 0.138 \\
\hline $\mathrm{PCl}$ & 77.4 & 73.5 & $1.08(1.016-1.150)$ & $0.014^{*}$ \\
\hline CABG & 7.6 & 4.7 & $1.46(1.313-1.626)$ & $<0.001^{*}$ \\
\hline Revascularization & 83.9 & 77.7 & $1.24(1.158-1.334)$ & $<0.001^{*}$ \\
\hline AKI & 15.6 & 15.7 & $1.10(1.013-1.193)$ & $0.023^{*}$ \\
\hline Electrical cardioversion/defibrillation & 4.6 & 4.0 & $1.18(1.041-1.326)$ & $0.009^{*}$ \\
\hline Cardiogenic shock & 11.5 & 13.3 & $0.91(0.837-0.992)$ & $0.032^{\star}$ \\
\hline
\end{tabular}




\section{Cureus}

\begin{tabular}{|c|c|c|c|c|}
\hline Outcome & Morbid obesity, \% & Nonobese, \% & aOR (95\% Cl) & p-value \\
\hline \multicolumn{5}{|l|}{ Primary outcome } \\
\hline In-hospital mortality & 7.8 & 8.4 & $1.26(1.100-1.446)$ & $0.001^{*}$ \\
\hline \multicolumn{5}{|l|}{ Secondary outcomes } \\
\hline Mean Length of stay, days $(95 \% \mathrm{Cl})$ & $4.7(4.5-4.9)$ & $4.0(4.0-4.1)$ & $0.34(0.170-0.516)$ & $<0.001^{*}$ \\
\hline Mean total hospital charges, US\$ (95\% Cl) & $120500(115900-125200)$ & $107600(105400-109800)$ & $7900(3900-12000)$ & $<0.001^{*}$ \\
\hline PCI with DES & 63.5 & 65.1 & $0.91(0.841-0.973)$ & $0.007^{*}$ \\
\hline PCl with BMS & 10.0 & 9.2 & $1.11(0.994-1.235)$ & 0.063 \\
\hline $\mathrm{PCl}$ & 72.5 & 73.5 & $0.92(0.846-0.991)$ & $0.030^{\star}$ \\
\hline CABG & 6.8 & 4.7 & $1.35(1.170-1.559)$ & $<0.001^{*}$ \\
\hline Revascularization & 78.8 & 77.7 & $0.99(0.911-1.086)$ & 0.905 \\
\hline AKI & 21.0 & 15.7 & $1.47(1.333-1.610)$ & $<0.001^{*}$ \\
\hline Electrical cardioversion/defibrillation & 5.2 & 4.0 & $1.31(1.128-1.526)$ & $<0.001^{\star}$ \\
\hline Cardiogenic shock & 13.6 & 13.3 & $1.05(0.944-1.160)$ & 0.383 \\
\hline
\end{tabular}

\section{TABLE 3: Clinical outcomes of STEMI in morbidly obese patients}

*: Statistically significant, AKI: Acute kidney failure, aOR: adjusted odds ratio, BMS: Bare metal stent, CABG: Coronary artery bypass grafting, CI: Confidence interval, DES: Drug eluting stent, PCI: Percutaneous coronary intervention, STEMI: ST segment elevation myocardial infarction.

\section{Secondary outcomes in STEMI patients}

Mild to moderately obese patients had higher odds of composite revascularization (aOR: 1.24, 95\% CI: 1.158 1.334, $\mathrm{p}<0.001$ ), PCI (aOR: 1.08, 95\% CI: 1.054-1.150, $\mathrm{p}=0.014$ ), and CABG (aOR: 1.46, 95\% CI: 1.313-1.626, $\mathrm{p}<0.001$ ). These patients had lower odds of cardiogenic shock (aOR: 0.91, 95\% CI: 0.837-0.992, $\mathrm{p}=0.032$ ), but higher odd of AKI (aOR: 1.10, 95\% CI: 1.013-1.193, $\mathrm{p}=0.023$ ) and electrical cardioversion or defibrillation (aOR: 1.18, 95\% CI: 1.041-1.326, $\mathrm{p}=0.009$ ) when compared to non-obese patients (Table 2).

There was no difference in rate of composite revascularization between morbidly obese and non-obese patients (aOR: 0.99, 95\% CI: 0.911-1.086, $\mathrm{p}=0.905$ ). Morbidly obese patients had lower odds of PCI (aOR: 0.92, 95\% CI: 0.846-0.991, $\mathrm{p}=0.030$ ), but higher odds of CABG (aOR: 1.35, 95\% CI: $1.170-1.559, \mathrm{p}<0.001$ ) and AKI (aOR: 1.47, 95\% CI: 1.333-1.610, p < 0.001) compared to non-obese patient (Table 3).

\section{Characteristics of NSTEMI patients}

Obese patients admitted for NSTEMI had a significantly lower mean age compared to non-obese patients (63.0 vs 69.5 years, $\mathrm{p}<0.001$ ). Obese patients had higher proportion of medical comorbidities (Table 1 ).

\section{Primary outcome in NSTEMI patients: in-hospital mortality}

A total of 949,985 hospitalizations involved patients with NSTEMI. The in-hospital mortality for patients with NSTEMI was $3.5 \%$ overall. Mild to moderately obese patients with NSTEMI had a lower adjusted odds ratio for mortality (aOR: $0.73,95 \% \mathrm{CI}: 0.660-0.811, \mathrm{p}<0.001$ ) when compared to non-obese patients with NSTEMI. Patients with morbid obesity had no difference in mortality (aOR: 0.95, 95\% CI: 0.854-1.055, p = 0.333) compared to non-obese patients (Tables 4, 5). 


\section{Cureus}

\begin{tabular}{|c|c|c|c|c|}
\hline Outcome & Mild-moderate, \% & Nonobese, \% & aOR (95\% Cl) & p-value \\
\hline \multicolumn{5}{|l|}{ Primary outcome } \\
\hline In-hospital mortality & 2.0 & 3.8 & $0.73(0.660-0.811)$ & $<0.001^{*}$ \\
\hline \multicolumn{5}{|l|}{ Secondary outcomes } \\
\hline Mean Length of stay, days $(95 \% \mathrm{Cl})$ & $4.8(4.7-4.9)$ & $4.5(4.4-4.5)$ & $0.28(0.205-0.351)$ & $<0.001^{*}$ \\
\hline Mean total hospital charges, US\$ ( $95 \%$ Cl) & $96400(94100-38800)$ & $83800(82200-85400)$ & $7100(5400-8700)$ & $<0.001^{\star}$ \\
\hline PCl with DES & 36.4 & 31.2 & $1.06(1.028-1.100)$ & $<0.001^{*}$ \\
\hline PCI with BMS & 3.2 & 3.2 & $0.97(0.887-1.051)$ & 0.420 \\
\hline $\mathrm{PCl}$ & 39.3 & 34.1 & $1.05(1.017-1.088)$ & $0.003^{\star}$ \\
\hline Revascularization & 54.1 & 42.3 & $1.31(1.262-1.350)$ & $<0.001^{\star}$ \\
\hline CABG & 15.0 & 8.3 & $1.65(1.576-1.747)$ & $<0.001^{*}$ \\
\hline AKI & 19.6 & 20.0 & $1.06(1.014-1.108)$ & $0.011^{*}$ \\
\hline Electrical cardioversion/defibrillation & 1.4 & 1.2 & $1.12(0.985-1.284)$ & 0.081 \\
\hline Cardiogenic shock & 3.2 & 3.6 & $0.93(0.855-1.016)$ & 0.110 \\
\hline
\end{tabular}

\section{TABLE 4: Clinical outcomes of NSTEMI in mild to moderately obese patients}

*: Statistically significant, AKI: Acute kidney failure, aOR: adjusted odds ratio, BMS: Bare metal stent, CABG: Coronary artery bypass grafting, Cl: Confidence interval, DES: Drug eluting stent, PCI: Percutaneous coronary intervention, NSTEMI: Non-ST segment elevation myocardial infarction. 


\section{Cureus}

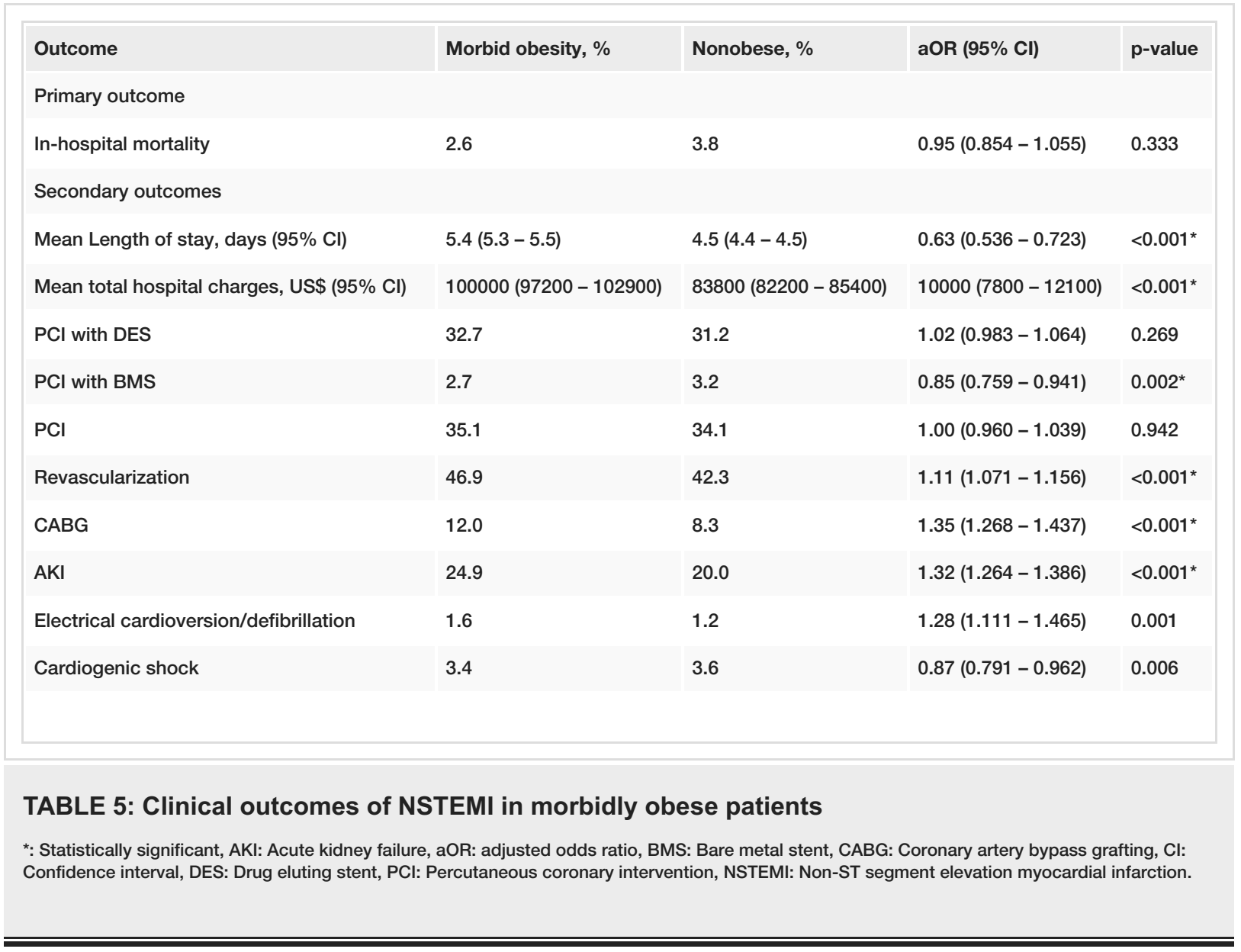

\section{Secondary outcomes in NSTEMI patients}

Mild to moderately obese patients with NSTEMI had significantly higher adjusted odds ratio of composite revascularization (aOR: 1.31, 95\% CI: 1.262-1.350, p < 0.001), PCI (aOR: 1.05, 95\% CI: 1.017-1.088, p = 0.003 ), and CABG (aOR: $1.65,95 \% \mathrm{CI}: 1.576-1.747, \mathrm{p}<0.001$ ). They also had longer length of hospitalization and higher total hospital charges compared to non-obese patients (Table 4).

Morbidly obese patients with NSTEMI had significantly higher adjusted odds ratio of composite revascularization (aOR: 1.11, 95\% CI: 1.071-1.156, p < 0.001) and CABG (aOR: 1.35, 95\% CI: 1.268-1.437, p < $0.001)$. They also had longer length of hospitalization and higher total hospital charges compared to nonobese patients (Table 5).

\section{Discussion}

Obesity is prevalent in patients with AMI. Although more males had AMI, obesity was associated with a higher prevalence in females with AMI. This is congruent with the overall higher prevalence of obesity in females in the US [3]. Obese patients were significantly younger on hospitalization in both the STEMI and NSTEMI groups, likely due to the association of obesity with early development of coronary disease [23, 24]. Whites, Blacks and Hispanics with AMI also had a higher proportion of obese patients suggesting that the racial disparity in obese population also reflects in these patients [3].

Among patients with STEMI, mild to moderately obese patients had lower odds of inpatient mortality. However, morbidly obese patients had higher odds of inpatient mortality compared to nonobese patients. This showed that the severity of obesity likely impacted mortality in STEMI patients. A conclusion reached by Das et al. showed that morbid obesity was independently associated with worse outcomes among patients with STEMI [9]. This finding is at variance with a study by Dhoot et al., which demonstrated lower odds of mortality in morbidly obese patients [25]. This study did not stratify AMI which could have been a confounding factor.

In NSTEMI, mild to moderately obese patients had a significantly lower adjusted odds for inpatient mortality compared to nonobese patients. There was no difference in mortality between morbidly obese patients and non-obese patients with NSTEMI.

The study demonstrated higher odds of composite revascularization in mild to moderately obese patients 


\section{Cureus}

with STEMI. There was no difference in composite revascularization between morbidly obese patients and non-obese patients. This finding may be associated with better outcomes in patients with mild-moderate compared to morbidly obese patients who had STEMI. Among patients with NSTEMI, both mild-moderate and morbidly obese patients had higher odds of revascularization compared to non-obese patients.

There was increasingly higher hospital resource utilization with levels of obesity. This is seen in the rising length of hospitalization and the total hospital charges. This is similar to a study by Champagne-Langabeer et al., which showed morbidly obese patients had longer treatment times compared to other patients with MI [26]. This places significant stress on the healthcare institutions amid limited resources.

Various reasons have been postulated for the better outcome in mild to moderately obese patients including lower incidence of undernutrition, weight loss, possible presence of protective cytokine, greater metabolic reserves and possibly differing obesity phenotypes [11,24]. We also suggest that although obesity is a risk factor for cardiovascular diseases, in patients with AMI, the earlier age of presentation as well as the statistically significant higher rates of interventions including PCI and CABG, procedures with known mortality benefits, help to offset this risk. However, the poorer outcomes in morbidly obese patients suggest that with progression of obesity, there is a proportional increase in cardiovascular risk.

Our study has some important limitations. NIS database is subject to non-randomization. The NIS is an administrative database that uses ICD-10 codes to characterize diagnoses and hospitalization events [27]. BMI could not be coded on a linear scale, as BMI ranges rather than individual BMI values are available. The disability associated with obesity could not be measured using the NIS database. Data in NIS is on hospitalizations, rather than individual patients. Hence if the same patient gets admitted on more than one occasion, that patient will be counted multiple time [28]. There is no reliable way to determine if secondary diagnoses preceded or started in the index hospitalization. NIS studies cannot establish causation, but only association.

\section{Conclusions}

The degree of obesity affects outcome of patients with AMI. Cardiovascular interventions during hospitalizations for AMI also varied with degree of obesity. This may have affected the outcome, especially among morbidly obese patients. The reasons for these differences are not clear. However, morbidly obese patients had poorer outcomes compared to patients with only mild-moderate obesity. Increased revascularization procedures may improve outcomes in obese patients. Further studies are required to elucidate factors responsible for this paradox as well as identifying the point at which these variables no longer improve outcomes in obese people.

\section{Appendices}

\begin{tabular}{|c|c|}
\hline & ICD-10 codes \\
\hline \multicolumn{2}{|l|}{$\begin{array}{l}\text { Dlagnosis } \\
\text { codes }\end{array}$} \\
\hline STEMI & $|21.0| 21.1,,|21.2| 21.3$, \\
\hline NSTEMI & 121.4 \\
\hline Obesity & E66.0, E66.1, E66.2, E66.8, E66.9, Z68.3, Z68.4 \\
\hline \multicolumn{2}{|c|}{$\begin{array}{l}\text { Procedure } \\
\text { codes }\end{array}$} \\
\hline PTCA & 02703ZZ, 02704ZZ, 02713ZZ, 02714ZZ, 02723ZZ, 02724ZZ, 02733ZZ, 02734ZZ \\
\hline PCI BMS & $\begin{array}{l}\text { 02703D6, 02703DZ, 02704D6, 02704DZ, 02703E6, 02703EZ, 02704E6, 02704EZ, 02703F6, 02703FZ, 02704F6, } \\
\text { 02704FZ, 02703G6, 02703GZ, 02704G6, 02704GZ, 02713D6, 02713DZ, 02714D6, 02714DZ, 02713E6, 02713EZ, } \\
\text { 02714E6, 02714EZ, 02713F6, 02713FZ, 02714F6, 02714FZ, 02713G6, 02713GZ, 02714G6, 02714GZ, 02723D6, } \\
\text { 02723DZ, 02724D6, 02724DZ, 02723E6, 02723EZ, 02724E6, 02724E6, 02724EZ, 02723F6, 02723FZ, 02724F6, } \\
\text { 02724FZ, 02723G6, 02723GZ, 02724G6, 02724GZ, 02733D6, 02733DZ, 02734D6, 02734DZ, 02733E6, 02733EZ, } \\
\text { 02734E6, 02734EZ, 02733F6, 02733FZ, 02733FZ, 02734F6, 02733G6, 02733GZ, 02734G6, 02734GZ }\end{array}$ \\
\hline PCI DES & $\begin{array}{l}\text { 0270346, 027034Z, 0270446, 027044Z, 0270356, 027035Z, 0270456, 027045Z, 0270366, 027036Z, 0270466, } \\
\text { 027046Z, 0270376, 027037Z, 0270476, 027047Z, 0271346, 027134Z, 0271446, 027144Z, 0271356, 027135Z, } \\
\text { 0271456, 027145Z, 0271366, 027136Z, 0270376, 0271466, 027146Z, 0271376, 027137Z, 0271476, 027147Z, } \\
\text { 0272346, 027234Z, 0272446, 027244Z, 0272356, 027235Z, 0272456, 027245Z, 0272366, 027236Z, 027246Z, } \\
\text { 0272376, 027237Z, 0272476, 027035Z, 027247Z, 0273346, 027334Z, 0273446, 027344Z, 0273356, 027335Z, } \\
\text { 0273456, 027345Z, 0273366, 027336Z, 0273466, 027346Z, 0273376, 027337Z, 027045Z, 0273476, 027347Z }\end{array}$ \\
\hline IABP & $5 \mathrm{~A} 02210,5 \mathrm{~A} 02110$ \\
\hline
\end{tabular}




\section{Cureus}

PEAD

02HA0RJ, 02HA3RJ, 02HA4RJ, 5A02116, 5A0211D, 5A02216, 5A0221D, 02HA3RZ, 5A02216

Intracoronary

artery

thrombolytic

3E07017, 3E07317

infusion

CABG

0210093, 0210098, 0210099, 021009C, 021009F, 021009W, 02100A3, 02100A8, 02100A9, 02100AC, 02100AF, 02100AW, 0211093, 0211098, 0211099, 021109C, 021109F, 021109W, 02110A3, 02110A8, 02110A9, 02110AC, 02110AF, 02110A, 0212093, 0212098, 0212099, 021209C, 021209F, 021209W, 02120A3, 02120A8, 02120A9, 02120AC, 02120AF, 02120AW, 0213093, 0213098, 0213099, 021309C, 021309F, 021309W, 02130A3, 02130A8, 02130A9, 02130AC, 02130AF, 02130AW

Comorbidities

Dyslipidemia E78

Old MI $\quad 1252$

Old PCl Z Z $\quad$ Z9861

Old CABG Z Z951

Old pacemaker $\quad$ Z950

Atrial

fibrillation/flutter 148

Chronic

obstructive

pulmonary J41, J42, J43, J44

disease

Old stroke $\quad 169$

Hypertension $\quad \mathbf{1 1 0}$

Peripheral

vascular

disease

Hypothyroidism E03

Diabetes

Mellitus Type E10, E11

$1 \& 2$

Congestive

heart Failure

150

Chronic Kidney

Disease

N18

Liver disease $\quad \mathrm{K} 70, \mathrm{~K} 71, \mathrm{~K} 72, \mathrm{~K} 73, \mathrm{~K} 74, \mathrm{~K} 75, \mathrm{~K} 76, \mathrm{~K} 77$

Electrolyte

derangement

E870, E871, E872, E873, E874, E875, E876

Oxygen

dependence

Z9981

Smoking Z87891, F17200

Anemia

D50, D51, D52, D53, D55, D56, D57, D58, D59, D60, D61, D62, D63, D64

\section{TABLE 6: Used ICD-10 codes}

ACS: Acute Coronary Syndrome, STEMI: ST Elevation Myocardial infarction, NSTEMI: Non-ST Elevation Myocardial Infarction, UA: Unstable Angina, RA: Rheumatoid Arthritis, MI: Myocardial Infarction, PCI: Percutaneous Coronary Intervention, CABG: Coronary Artery Bypass Graft, PTCA: Percutaneous Transluminal Coronary Angioplasty, PCI DES: Percutaneous Coronary Intervention with Drug Eluting Stent, PCI BMS: Percutaneous Coronary Intervention with Bare Metal Stent, IABP: Intra-aortic Balloon Pump, PEAD: Percutaneous External Assist Devices. 


\section{Additional Information \\ Disclosures}

Human subjects: Consent was obtained by all participants in this study. N/A issued approval N/A. The NIS database does not contain patient identifiers. Since 2012, the NIS has also removed state level and hospital identifiers. This has enhanced patient protection and anonymity. This study was exempt from Institutional Review Board approval. Animal subjects: All authors have confirmed that this study did not involve animal subjects or tissue. Conflicts of interest: In compliance with the ICMJE uniform disclosure form, all authors declare the following: Payment/services info: All authors have declared that no financial support was received from any organization for the submitted work. Financial relationships: All authors have declared that they have no financial relationships at present or within the previous three years with any organizations that might have an interest in the submitted work. Other relationships: All authors have declared that there are no other relationships or activities that could appear to have influenced the submitted work.

\section{References}

1. Ogden CL, Carroll MD, Fryar CD, Flegal KM: Prevalence of obesity among adults and youth: United States, 2011-2014. NCHS Data Brief. 2015, 1-8.

2. Hales CM, Fryar CD, Carroll MD, Freedman DS, Ogden CL: Trends in obesity and severe obesity prevalence in US youth and adults by sex and age, 2007-2008 to 2015-2016. JAMA. 2018, 319:1723-1725. 10.1001/jama.2018.3060

3. NHLBI Obesity Education Initiative Expert Panel on the Identification, Evaluation, and Treatment of Obesity in Adults (US): Clinical Guidelines on the Identification, Evaluation, and Treatment of Overweight and Obesity in Adults--The Evidence Report. National Heart, Lung, and Blood Institute, Bethesda; 1998.

4. Shaka H, Edigin E, Raghavan S, Gomez TM: The obesity paradox among patients hospitalized for bacterial pneumonia: outcomes of the nationwide inpatient sample. Chest. 2020, 158:A335. 10.1016/j.chest.2020.08.332

5. Bays HE, González-Campoy JM, Bray GA, et al.: Pathogenic potential of adipose tissue and metabolic consequences of adipocyte hypertrophy and increased visceral adiposity. Expert Rev Cardiovasc Ther. 2008, 6:343-368. 10.1586/14779072.6.3.343

6. Thygesen K, Alpert JS, Jaffe AS, et al.: Fourth universal definition of myocardial infarction (2018). J Am Coll Cardiol. 2018, 72:2231-2264. 10.1016/j.jacc.2018.08.1038

7. Sakr Y, Madl C, Filipescu D, et al.: Obesity is associated with increased morbidity but not mortality in critically ill patients. Intensive Care Med. 2008, 34:1999-2009. 10.1007/s00134-008-1243-0

8. Yaegashi M, Jean R, Zuriqat M, Noack S, Homel P: Outcome of morbid obesity in the intensive care unit . J Intensive Care Med. 2005, 20:147-154. 10.1177/0885066605275314

9. Das SR, Alexander KP, Chen AY, et al.: Impact of body weight and extreme obesity on the presentation, treatment, and in-hospital outcomes of 50,149 patients with ST-Segment elevation myocardial infarction: results from the NCDR (National Cardiovascular Data Registry). J Am Coll Cardiol. 2011, 58:2642-2650. 10.1016/j.jacc.2011.09.030

10. Diercks DB, Roe MT, Mulgund J, et al.: The obesity paradox in non-ST-segment elevation acute coronary syndromes: results from the Can Rapid risk stratification of Unstable angina patients Suppress ADverse outcomes with Early implementation of the American College of Cardiology/American Heart Association Guidelines Quality Improvement Initiative. Am Heart J. 2006, 152:140-148. 10.1016/j.ahj.2005.09.024

11. Vecchié A, Dallegri F, Carbone F, et al.: Obesity phenotypes and their paradoxical association with cardiovascular diseases. Eur J Intern Med. 2018, 48:6-17. 10.1016/j.ejim.2017.10.020

12. Lavie CJ, Milani RV, Ventura HO: Obesity and cardiovascular disease: risk factor, paradox, and impact of weight loss. J Am Coll Cardiol. 2009, 53:1925-1932. 10.1016/j.jacc.2008.12.068

13. Healthcare Cost and Utilization Project. Introduction to the HCUP National Inpatient Sample (NIS) . (2017). Accessed: December 04, 2020: https://www.hcup-us.ahrq.gov/db/nation/nis/NIS_Introduction_2017.jsp.

14. HCUP methods series. (2020). Accessed: December 04, 2020: http://www.hcupus.ahrq.gov/reports/methods/methods.jsp.

15. Edigin E, Rivera Palon MM: LB934 Outcomes of psoriasis with and without joint involvement. J Invest Dermatol. 2020, 140:8. 10.1016/j.jid.2020.05.026

16. Edigin E, Prado V, Shaka H, Salazar M: Lung involvement in systemic lupus erythematosus increases inpatient mortality: analysis of the national inpatient sample. Chest. 2020, 158:A1871. 10.1016/j.chest.2020.08.1623

17. Edigin E, Eseaton P, Kaul S, et al.: Systemic sclerosis is not associated with worse outcomes of patients admitted for ischemic stroke: analysis of the national inpatient sample. Cureus. 2020, 12:9155. 10.7759/cureus.9155

18. Edigin E, Ojemolon PE, Eseaton PO, et al.: Rheumatoid arthritis patients have better outcomes when hospitalized for ischemic stroke: analysis of the national inpatient sample (PREPRINT). J Clin Rheumatol. 2020, 10.1097/RHU.0000000000001563

19. Edigin E, Akuna E, Asemota I, Eseaton P, Ojemolon PE, Shaka H, Manadan A: Rheumatoid arthritis does not negatively impact outcomes of patients admitted for atrial fibrillation. Cureus. 2020, 12:10241. 10.7759/cureus.10241

20. Ojemolon PE, Shaka H, Edigin E, et al.: Impact of diabetes mellitus on outcomes of patients with knee osteoarthritis who underwent knee arthroplasty: an analysis of the nationwide inpatient sample. Cureus. 2020, 12:8902. 10.7759/cureus.8902

21. Edigin E, Shaka H, Eseaton P, et al.: Rheumatoid arthritis is not associated with increased inpatient mortality in patients admitted for acute coronary syndrome. Cureus. 2020, 12:9799. 10.7759/cureus.9799 


\section{Cureus}

22. Edigin E, Ojemolon PE, Eseaton PO, Shaka H, Akuna E, Asemota IR, Manadan A: Systemic sclerosis is associated with increased inpatient mortality in patients admitted for atrial fibrillation: analysis of the national inpatient sample (PREPRINT). J Clin Rheumatol. 2020, 10.1097/RHU.0000000000001543

23. Kachur S, Lavie CJ, de Schutter A, Milani RV, Ventura HO: Obesity and cardiovascular diseases. Minerva Medica. 2017, 108:212-228. 10.23736/S0026-4806.17.05022-4

24. Lavie CJ, De Schutter A, Parto P, et al.: Obesity and prevalence of cardiovascular diseases and prognosis The obesity paradox updated. Progress Cardiovasc Dis. 2016, 58:537-547. 10.1016/j.pcad.2016.01.008

25. Dhoot J, Tariq S, Erande A, Amin A, Patel P, Malik S: Effect of morbid obesity on in-hospital mortality and coronary revascularization outcomes after acute myocardial infarction in the United States. Am J Cardiol. 2013, 111:1104-1110. 10.1016/j.amjcard.2012.12.033

26. Champagne-Langabeer T, Kim J, Bower JK, Gardner A, Fowler R, Langabeer JR 2nd: Obesity, treatment times, and cardiovascular outcomes after ST-elevation myocardial infarction: findings from mission: Lifeline North Texas. J Am Heart Assoc. 2017, 6:e005827. 10.1161/JAHA.117.005827

27. Edigin E, Kaul S, Eseaton PO: Analysis of hidradenitis suppurativa hospitalizations: a report from the National Inpatient Sample database (IN PRESS). J Am Acad Dermatol. 2020, 10.1016/j.jaad.2020.10.083

28. Jamal S, Khan MZ, Kichloo A, et al.: The effect of atrial fibrillation on inpatient outcomes of patients with acute pancreatitis: a two-year national inpatient sample database study. J Innov Cardiac Rhythm Manage. 2020, 12:1-6. 10.19102/icrm.2021.120102 